\title{
Research on the Application of Inverse Fuzzy Number to the Time Series Forecasting Algorithm of Tourism Revenue
}

\author{
Pingli $\mathrm{Xu}^{1, \mathrm{a}}$, Yufei Wang ${ }^{1, \mathrm{~b}}$ and Kun Zhang ${ }^{1, \mathrm{c}^{*}}$ \\ ${ }^{1}$ College of Computer Engineering, Hainan Tropical Ocean University, Sanya, Hainan, 572022, China \\ a51387512@qq.com, b1576949369@qq.com, 'zk0588@163.com \\ *The corresponding author
}

Keywords: Model time series prediction; Percentage; Formula modeling; Inverse fuzzy number; Total tourism income.

\begin{abstract}
A kind of improved method, applied to Hangzhou Songcheng theme park tourism income and found that are known in the simulation and prediction of the value, regional value of grey model to predict the smaller fuzzy time series forecasting is proposed in this paper. The prediction formula is simple and convenient, and it is easy to calculate. The error rate of historical simulation is small, and the main idea of forecasting data is a kind of method which can be trusted.
\end{abstract}

\section{Introduction}

Random, lack of relevant parameters, widespread imprecise information and other factors, the stochastic theory is difficult to effectively solve the tourism revenue forecasting problems. The tourism literature [1] revenue forecast problem is considered as a grey system, the application of GM $(1,1)$ grey model prediction of Hangzhou Songcheng theme park tourism income, can not only predict the history the data, but also for the short and medium term unknown year data prediction is feasible method to research this problem. Song $[2,3,4]$ took the college entrance rate as fuzzy system, said university enrollment data by fuzzy language, fuzzy time series is proposed for the first time, and proposes a fuzzy time series model is applied to prediction of University of Alabama admission rate. The frequency domain based on density divided by literature [5], and first proposed the concept of fuzzy number inverse The application of the model proposed by University of Alabama, enrollment rate prediction for the smaller $0.57 \%$; literature improved [6] literature [5] model, has the same problem as small $0.47 \%$; [7] further improved the model of literature, has the same problem as $0.34 \%$ smaller, has a very high accuracy. But these models do not for the prediction of the unknown mentioned years of data. The [8] does not use [5,6,7] to document the method for domain partition, but directly use the historical data of the year by year and year by year percentage percentage difference, set up a domain, the inverse fuzzy number concept should paste use literature [5,6,7], reestablishing fuzzy time series prediction formula. A new prediction model of NFTSFM (New Fuzzy Time Series Forecasting Model), the same problem is very small $0.27 \%$, not only the history Data simulation and prediction precision is high, and NFTSFM literature [8] to predict unknown data. This paper on reference [8] method for small modifications, improved fuzzy time series forecasting model IFTSFM (improved fuzzy time series forecasting model) is proposed. IFTSFM re research in literature [1] using GM $(1,1)$ grey model of Hangzhou Songcheng theme park tourism revenue forecast, the historical data to predict the, IFTSFM than GM $(1,1)$ model smaller. When applied to predict unknown data and forecasting data of IFTSFM need decision to participate in and embodies the dominant ideology.

\section{Basic Concept}

Definition1. If the time series data is represented by fuzzy data, then it is called fuzzy time series.

Definition2. Set the real number set $U=\{U 1, U 2, \ldots, U n\}$ and $W 2, W=\{W 1, \ldots, W m\}$ is the 2 domain. 


$$
A_{i}=\frac{0.006}{U_{i}}+\frac{1}{U_{i}+\mathrm{W}_{j}}, \quad(j \in\{1,2, \mathrm{~L}, m\})
$$

It is known as the $\mathrm{U}$ and $\mathrm{W}$ on the number of fuzzy.

Definition3. Set the real number set $U=\{U 1, U 2, \ldots, U n\}$ and $W 2, W=\{W 1, \ldots, W m\}$ is the 2 domain.

$$
B_{i}=\frac{1.006}{\frac{0.006}{U_{i}}+\frac{1}{U_{i}+\mathrm{W}_{j}}}, \quad(j \in\{1,2, \mathrm{~L}, m\})
$$

It is known as the inverse fuzzy number corresponding to $\mathrm{U}$ and $\mathrm{W}$.

Definition4. Annual count of tourism revenue series I2, I1, . , In, year by year as a percentage of

$$
U_{i}=\left[\left(I_{i}-I_{i-1}\right) \div I_{i-1}\right] \times 100 \%
$$

Definition5. Annual count of tourism revenue series $\mathrm{I} 2, \mathrm{I} 1, \ldots, \mathrm{Fi}$, In is a predictive value, and the prediction error rate is defined as

$$
\left|I_{i}-F_{i}\right| \div I_{i}
$$

Average Forecasting Error Rate is:

$$
A F E R=\frac{1}{n} \sum_{i=1}^{n}\left(\left|I_{i}-F_{i}\right| \div I_{i}\right) \times 100 \%
$$

\section{The Concrete Application Steps of IFTSFM}

Step 1 set up 3 domains. $\mathrm{I}=\{\mathrm{I} 1, \mathrm{I} 2, \ldots$, In $\}$, tourism revenue year by year percentage of the domain $\mathrm{U}=\{\mathrm{U} 2, \mathrm{U} 3, \ldots, \mathrm{Un}\}$ and the percentage of piecewise domain $\mathrm{W}=\{\mathrm{W} 1, \mathrm{~W} 2, \ldots \mathrm{Wm}\}$

Step2 establish the prediction formula of IFTSFM.

Step3 apply IFTSFM to predict the study.

\section{Prediction of Tourism Revenue of Songcheng Theme Park in Hangzhou}

In the literature [1] gives the tourism income of Songcheng Hangzhou theme park as shown in Table 1.

Table 1 Songcheng Hangzhou theme park 2007 2012 annual tourism income

\begin{tabular}{|c|rrrrrr|}
\hline Year & 2007 & 2008 & 2009 & 2010 & 2011 & 2012 \\
\hline Tourism revenue (10000 yuan) & 11724.3 & 14167.6 & 18759.6 & 32101.4 & 34123.4 & 39499.1 \\
\hline
\end{tabular}

From Table 1 to get the tourism revenue:

$\mathrm{I}=\{\mathrm{I} 2007=11724.3, \mathrm{I} 2008=14167.6, \mathrm{I} 2009=18759.6$,

$\mathrm{I} 2010=32101.4, \mathrm{I} 2011=34123.4, \mathrm{I} 2012=39499.1\}$.

To set up the percentage of year by year.

Application formula (3), the Songcheng theme park tourism revenue year by year percentage, as shown in Table 2.

Table 2 from the Songcheng theme park tourism revenue year by year percentage of the area: 
$\mathrm{U}=\{\mathrm{U} 2008=20.84, \mathrm{U} 2009=32.41, \mathrm{U} 2010=71.12, \mathrm{U} 2011=6.30, \mathrm{U} 2012=15.75\}$.

Set up a percentage of the section on the domain. Max\{U2008, U2009, U2010, U2011, $\mathrm{U} 2012\} / 5=71.12 / 10=7.112$. Get the percentage of subsection

$$
\begin{aligned}
W= & \left\{W_{-10}=-71.12, W_{-9}=-64.008, W_{-8}=-56.896, W_{-7}=-49.784,\right. \\
& W_{-6}=-42.672, W_{-5}=-35.56, W_{-4}=-28.448, W_{-3}=-21.336, \\
& W_{-2}=-14.224, W_{-1}=-7.112, W_{0}=0, W_{1}=7.112, W_{2}=14.224, \\
& W_{3}=21.336, W_{4}=28.448, W_{5}=35.56, W_{6}=42.672, \\
& \left.W_{7}=49.784, W_{8}=56.896, W_{9}=64.008, W_{10}=71.12\right\}
\end{aligned}
$$

Establish the prediction formula of IFTSFM

Define 6 prediction formula as

$$
B_{i}=\frac{0.006+1}{\frac{0.006}{U_{i-1}}+\frac{1}{U_{i-1}+W_{j}}}, \quad F_{i}=I_{i-1} \times\left(1+B_{i} \div 100\right) \text {, }
$$

Among them, $U_{i-1} \in U$ is an element in the percentage of year by year (6), $W_{j} \in W$ is the element in the percentage of subsection theory domain (7), and $I_{i-1} \in I$ is the element of tourism income theory domain (5).

Application prediction formula (8), the Songcheng theme park 2007 2012 years of tourism revenue forecast research.

$U_{i-1} \in U$ piecewise prediction in 200921020112012 years minimum in the formula (8) in a, and by formula (6) know $U_{2008}=20.84$ is the yearly percentage of domain data. Therefore, in the application of prediction formula (8) on the song Cheng theme park from 2007 to 2012 tourism income prediction research, can be predicted only from 2009 forecast calculation, which can only calculate data. For them each year, as the percentage of domain (7) $21 \mathrm{w}_{\mathrm{i}}$ value. Each take a $\mathrm{w}_{\mathrm{i}}$ value prediction formula (8) can be calculated in the forecasting data, so the introduction of the following definite meaning.

Define 7 of all forecast data in a given year of known data, and historical data nearest to the known data of the forecast data is called the year's forecast data.

The definition of 7 can greatly simplify the calculation process of artificial calculation. For example:

$$
\begin{aligned}
& B_{2009}=\frac{0.006+1}{\frac{0.006}{U_{2008}}+\frac{1}{U_{2008}+\mathrm{W}_{1}}}=\frac{1.006}{\frac{0.006}{20.84}+\frac{1}{20.84+7.112}}=\frac{1.006}{\frac{0.006}{20.84}+\frac{1}{27.952}} \\
& =\frac{1.006}{0.000287908+0.035775615}=\frac{1.006}{0.036063523}=27.89522255 \\
& F_{2009}=I_{2008} \times\left(1+B_{2009} \div 100\right)=14167.6 \times 1.2789522255=18119.7 \\
& \left|I_{2009}-F_{2009}\right| / I_{2009}=|18119.7-18759.6| / 18759.6=0.034111 \\
& B_{2009}=\frac{0.006+1}{\frac{0.006}{U_{2008}}+\frac{1}{U_{2008}+\mathrm{W}_{2}}}=\frac{1.006}{\frac{0.006}{20.84}+\frac{1}{20.84+14.224}}=\frac{1.006}{\frac{0.006}{20.84}+\frac{1}{35.064}} \\
& =\frac{1.006}{0.000287908+0.028519279}=\frac{1.006}{0.028807187}=34.92184086 \\
& F_{2009}=I_{2008} \times\left(1+B_{2009} \div 100\right)=14167.6 \times 1.3492184086=19115.2 \\
& \left|I_{2009}-F_{2009}\right| / I_{2009}=|19115.2-18759.6| / 18759.6=0.018955
\end{aligned}
$$




$$
\begin{aligned}
& B_{2009}=\frac{0.006+1}{\frac{0.006}{U_{2008}}+\frac{1}{U_{2008}+\mathrm{W}_{3}}}=\frac{1.006}{\frac{0.006}{20.84}+\frac{1}{20.84+21.336}}=\frac{1.006}{\frac{0.006}{20.84}+\frac{1}{42.176}} \\
& =\frac{1.006}{0.000287908+0.023998074}=\frac{1.006}{0.024285982}=41.42307278 \\
& F_{2009}=I_{2008} \times\left(1+B_{2009} \div 100\right)=14167.6 \times 1.4142307278=20036.3 \\
& \left|I_{2009}-F_{2009}\right| / I_{2009}=|20036.3-18759.6| / 18759.6=0.068056
\end{aligned}
$$

Thus obtained in 2009 forecast data for $\mathrm{F}_{2009}=19115.2$, the parameters used $\mathrm{U}_{2008}+\mathrm{W}_{2}$, and $\left|I_{i}-F_{i}\right| \div I_{i}=0.018955$. Results fill in the Table 2. Now is applied in the prediction of equation (8) to calculate the 201020112012 in each of the forecast data, results are still filled with such as Table 2. Calculated average prediction error rate of $A F E R=1.69 \%$.

\section{Summary}

Song Cheng theme park built from so far, there is no after market cultivation period, but directly enter a period of rapid growth. Illustrate the idea of the project is very novel, and tourists demand extremely fit, get people enthusiastic participation. But uncertainty about the ups and downs of the growth in the number of tourists, to the income prediction difficult.

IFTSFM formula for the prediction of the structure is extremely simple, is not only simple in calculation, and the computation is small. Especially when the expected to happen and has happened significant positive or major bad, adjusting the segment on the value of the elements in the domain can be quickly calculated may alter the forecast data to decision makers to make quick decisions.

IFTSFM a short-term prediction model, the effectiveness better. Due to historical data simulation to predict the average prediction error rate $A F E R$ is very small makes people to bring to the subject in the prediction accuracy of the prediction data about the unknown in a good expected by application of IFTSFM. Especially for belonging to the application of $\operatorname{GM}(1,1)$ model subject to historical data simulation and prediction of prediction error is large (for example AFER more than 5\%) when, you can consider the use of IFTSFM.

\section{Acknowledgements}

The work was supported by the 2014 innovation and entrepreneurship training college national college projects (No. 201411100068).

\section{References}

[1] Lin Cheng, Qian Cheng, Research on the prediction of tourist quantity and economic income of Hangzhou theme park based on Grey Model, Economic Research Guide, No.15, pp. 223-225, 2014.

[2] Song Q, Chissom B S, Forecasting enrollments with fuzzy time series--Part I, Fuzzy Sets and Systems, No.54, pp. 1-9, 1993.

[3] Song Q, Chissom B S, Forecasting enrollments with fuzzy time series, Fuzzy Sets and Systems, No.54, pp. 269-277, 1993.

[4] Song Q, Chissom B S, Forecasting enrollments with fuzzy time series-Part II, Fuzzy Sets and Systems, No.62, pp. 1-8, 1994.

[5] T A Jilani, S M A Burney, C Ardil, Fuzzy metric approach for fuzzy time series forecasting based on frequency density based partitioning, World Academy of Science, Engineering and Technology, No.34, pp. 1-6, 2007. 
[6] Stevenson M, Porter J E, Fuzzy time series forecasting using percentage change as the universe of discourse, World Academy of Science, Engineering and Technology, No.55, pp. 154-157, 2009.

[7] P Saxena, K Sharma, S Easo, Foreeca enrollment based on fuzzy time series with higher forecast accuracy rate, International Journal of Computer Technology and Applications, Vol.3, No.3, pp. 957-961, 2012.

[8] Hongxu Wang, Jianchun Guo, Hao Feng, Hailong Jin, A new forecasting model of fuzzy time series, Applied Mechanics and Materials, Vol.678, pp. 59-63, 2014.

[9] Pingli Xu, Tingting Zhang, Kun Zhang, Hongxu Wang, A new method based on intuitionistic fuzzy forecast of tourism revenue in Songcheng Hangzhou theme park, Economic Research Guide, No.7, pp. 159-163, 2016.

[10] Minsheng Liao, Kun Zhang, Hongxu Wang, Forecasting the total tourism revenue in Sanya based on the fuzzy time series model, Economic Research Guide, No.4, pp. 237-242, 2015. 